\title{
Neuronal avalanches change from wakefulness to deep sleep - a study of intracranial depth recordings in humans
}

\author{
Viola Priesemann ${ }^{1,2^{*}}$, Mario Valderrama ${ }^{3}$, Michael Wibral ${ }^{4}$, Michel Le Van Quyen ${ }^{5}$ \\ From Twenty Second Annual Computational Neuroscience Meeting: CNS*2013 \\ Paris, France. 13-18 July 2013
}

Neuronal dynamics differs between wakefulness and sleep stages, so does the cognitive state [1]. In contrast, a single attractor state, called self-organized critical (SOC), has been proposed to govern human brain dynamics for its optimal information coding and processing capabilities [2]. Here we address two open questions: First, does the human brain always operate in this computationally optimal state, even during deep sleep? Second, previous evidence for SOC was based on activity within single brain areas [3-5], however, the interaction between brain areas may be organized differently. Here we asked whether the interaction between brain areas is SOC.

We addressed these questions by characterizing neuronal avalanches [3] - spatiotemporal waves of enhanced activity - from up to 61 local field potential (LFP) channels of intracranial depth recordings (5 human patients, two recording nights each, summing up to $\sim 100 \mathrm{~h}$ of recordings). The recording contacts were distributed inside the entire brain. Note that $\sim 60$ contacts are sufficient to avoid major subsampling effects: Subsampling may heavily distort results in SOC systems due to an insufficient number of sampling sites [5]. In addition, we compared the experimental results to results from a subsampled SOC model of integrate- and fire neurons, which can be tuned to the sub- and supercritical regime.

We show that avalanche distributions closely follow a power law - the hall mark feature of SOC systems. This result held for each vigilance state, and independent of the threshold and the temporal scale. This indicates first that the interaction between brain areas are close to SOC, and second that the dynamics of all cognitive

\footnotetext{
* Correspondence: viola.priesemann@brain.mpg.de

${ }^{1}$ Max Planck Institute for Brain Research, Frankfurt, 60486, Germany

Full list of author information is available at the end of the article
}

states, from wakefulness to deep sleep are close to SOC. Minor differences between cognitive states are, however, reflected in the avalanche distributions: Slow wave sleep (s3/s4) showed larger and longer neuronal avalanches than REM sleep, while wakefulness showed intermediate ones $(p<0.05)$. The SOC neuronal model, together with the data, suggested first that these differences are mediated by global but tiny changes in synaptic strength, and second, that the changes with vigilance states reflect small deviations from criticality to the subcritical regime, implying that the human brain does not operate in the SOC state proper - contrary to previous believes. Independent of criticality, the analysis confirmed that slow wave sleep shows increased correlations between cortical areas, and revealed that REM sleep shows more fragmented cortical dynamics.

\section{Acknowledgements \\ VP received support from the Max Planck Society. VP and MW received support from LOEWE Grant Neuronale Koordination Forschungsschwerpunkt Frankfurt (NeFF). MV and MLVQ received support from the European Union- FP7 Project EPILEPSIAE (Evolving Platform for Improving Living Expectation of Subjects Suffering from IctAl Events, Grant No 211713). \\ Author details \\ ${ }^{1}$ Max Planck Institute for Brain Research, Frankfurt, 60486, Germany. ${ }^{2}$ Frankfurt Institute for Advanced Studies, Frankfurt, 60438, Germany. \\ ${ }^{3}$ University of Los Andes, Bogotá, Colombia. ${ }^{4}$ Goethe University, MEG Unit, Frankfurt, 60325, Germany. ${ }^{5}$ Hôpital de la Pitié-Salpêtrière, Paris, 75013, France. \\ Published: 8 July 2013

\author{
References \\ 1. Tononi G, Cirelli C: Sleep function and synaptic homeostasis. Sleep Med \\ Rev 2006, 10:49-62. \\ 2. Bertschinger $N$, Natschläger T: Real-Time Computation at the Edge of \\ Chaos in Recurrent Neural Networks. Neural Computation 2004, \\ 16:1413-1436.
}


3. Beggs JM, Plenz D: Neuronal avalanches in neocortical circuits. J Neurosci 2003, 23:11167-11177.

4. Ribeiro TL, Copelli M, Caixeta F, Belchior H, Chialvo DR, Nicolelis MAL, Ribeiro S: Spike Avalanches Exhibit Universal Dynamics across the SleepWake Cycle. PLOS ONE 2010, 5:e14129.

5. Priesemann V, Munk MHJ, Wibral M: Subsampling effects in neuronal avalanche distributions recorded in vivo. BMC Neurosci 2009, 10:40.

doi:10.1186/1471-2202-14-S1-P237

Cite this article as: Priesemann et al: Neuronal avalanches change from wakefulness to deep sleep - a study of intracranial depth recordings in humans. BMC Neuroscience 2013 14(Suppl 1):P237.

Submit your next manuscript to BioMed Central and take full advantage of:

- Convenient online submission

- Thorough peer review

- No space constraints or color figure charges

- Immediate publication on acceptance

- Inclusion in PubMed, CAS, Scopus and Google Scholar

- Research which is freely available for redistribution

Submit your manuscript at www.biomedcentral.com/submit 